Point of view

\title{
Swallowing improvement after levodopa treatment in idiopathic Parkinson's disease: Lack of evidence
}

\author{
Ailton Melo, Larissa Monteiro* \\ Division of Neurology and Epidemiology, Federal University of Bahia, Salvador-Bahia, Brazil
}

\section{A R T I C L E I N F O}

\section{Article history:}

Received 12 July 2012

Accepted 12 November 2012

\section{Keywords:}

Parkinson's disease

Levodopa

Dysphagia

Swallowing impairment

Non-motor symptoms

\begin{abstract}
A B S T R A C T
Swallowing impairment is the main cause of pulmonary infection and death in Parkinson's disease (PD). To date, there is no established effective treatment to restore swallowing function among these subjects. Levodopa is considered the gold standard treatment for motor symptoms in PD but its efficacy in improving oropharyngeal and esophageal dysphagia remains controversial.
\end{abstract}

(c) 2012 Elsevier Ltd. All rights reserved.

\section{Introduction}

Normal swallowing is a complex and coordinated physiological behavior consisting of a sequence of events responsible for transporting the food bolus from the mouth to the stomach and for maintaining airway protection from food residues [1].

Dysphagia is defined as impairment in swallowing liquids, solids or medications that occurs in any phase of deglutition. Swallowing impairment in persons with Parkinson's disease (PD) may interfere with nutritional status and predispose to pulmonary infection due to airway aspiration [2]. These events lead to increased morbidity, poor quality of life and decreased self-esteem.

Swallowing involves voluntary and reflex events. Optimal intraoral bolus formation (oral phase) requires adequate chewing of the food, closure of the lips, and coordinated masticatory and tongue movements that ensure effective jaw closing and bolus propulsion toward the posterior segment of the oral cavity, triggering the pharyngeal swallow reflex (pharyngeal phase). As in the oral phase, pharyngeal events require proper hyoid bone elevation, folding down of epiglottis in order to protect the airway, and cricopharyngeus relaxation, which allows transport of the food bolus into the esophagus (esophageal phase). After upper esophageal sphincter (UES) relaxation, peristaltic movements direct the bolus towards the stomach. These events are triggered by pattern generators within the medulla [3] and the coordinated sequence of

\footnotetext{
* Corresponding author. Rua Padre Feijo, 240, Quarto Andar, Divisão de Neurociências, Canela, Salvador-Bahia 40110-170, Brazil. Tel./fax: +55 7132476982.

E-mail address: menezes.lari@gmail.com (L. Monteiro).
}

events that culminates in deglutition depends on the integrity of neuronal pathways under the control of the hypoglossal nucleus, the nucleus of the solitary tract, and the nucleus ambiguus [4].

Reflex and voluntary cough are essential mechanisms of airway protection during swallowing, especially when food penetration occurs. However, previous data suggest that PD subjects have reduced laryngeal sensitivity and impaired cough reflex, making them silent aspirators [5,6]. Impairments in some deglutition events are responsible for the high prevalence of airway penetration and silent food or saliva aspiration in these patients [7]. Uncoordinated tongue movements and poor bolus formation, inefficient vallecula and pyriform fossa residue cleaning, incomplete laryngeal elevation, prolonged pharyngeal transit time (PPT), impaired relaxation of the upper esophageal sphincter, decreased esophageal peristaltic movements and lack of coordination between breathing and swallowing are the principal events that allow pharyngeal residue to enter the unprotected airway in PD patients [8].

The poor correlation between reported symptoms and objective findings during instrumental assessment of dysphagia in PD hampers early identification of penetration/aspiration; the consequent increased prevalence of silent food and saliva aspiration is responsible for the increased risk of pulmonary infection and higher mortality rates among persons with PD $[9,10]$. The reported prevalence of dysphagia in PD patients varies widely; however, a recent systematic review showed that PD patients are three times more likely than normal controls to develop oropharyngeal dysphagia [11]. This supports the idea that swallowing issues in PD deserve greater attention from both clinicians and researchers. 


\section{Levodopa treatment and swallowing improvement in Parkinson's disease: actual evidence}

Levodopa has been considered the gold standard treatment for PD since its introduction in the late 1960s. Cardinal motor symptoms unquestionably are relieved by levodopa administration. However, the efficacy of levodopa in alleviating autonomic symptoms, such as respiratory and gastrointestinal dysfunction, is still a matter of debate. In his 1817 seminal description of what we now know as PD, James Parkinson included some gastrointestinal features, such as sialorrhea and impairment in swallowing muscles [12]. In 1970, Calne et al. first reported that the duration of pharyngeal deglutition assessed by cineradiography in 18 parkinsonian subjects was not significantly altered by levodopa therapy [13].

\section{- Oral phase}

Abnormal events in the oral phase of swallowing have been reported previously in individuals with PD and may contribute to food aspiration and pulmonary infection. Employing the modified barium swallow (MBS) study, Hunter et al. studied 15 PD patients with symptomatic dysphagia and reported an unexpected increase in total oral transit time (OTT) for solids following levodopa administration, although fewer swallow attempts were needed to clear solid bolus [14]. Fuh et al. analyzed 15 PD patients by videofluoroscopy; 12 presented with swallowing impairment. Of these, half showed no improvement after levodopa use. After stratifying for deglutition phases, the most frequently altered events were in the oral phase (prolonged transit time, tongue elevation preventing bolus movement into the pharynx, oral tremor and reduced anterior-posterior tongue motion) [15]. Nagaya et al., in a similar study with 16 PD patients experiencing problems swallowing, detected an increased frequency of aspiration, as assessed by MBS, in subjects with tongue residue and repeated tongue pumping motion, compared with normal controls [16].

Motor fluctuations associated with long term levodopa therapy may contribute to swallowing impairment in PD patients. Monte et al. compared the OTT, as assessed by videofluoroscopy, between controls and both dyskinetic and nondyskinetic PD patients. There was no significant difference in OTT among the three groups [17]. Tongue bradykinesia may impair food transportation in the oral phase of deglutition. However, the total oral phase score does not correlate with speed of tongue movement but with the speed of mandibular movement, eliciting the hypothesis that the oral phase of swallowing may be responsive to dopaminergic stimulation, as these events are under voluntary control [18]. In a pilot study with 10 PD patients that investigated the integration between swallowing and respiration before and after levodopa use, the authors suggested that the risk of laryngeal penetration and tracheal aspiration in qualitative assessments of swallowing remained unchanged. They also reported that there were no differences in number of swallows, time taken per swallow, average time per swallow and swallowing capacity between "on" and "off" levodopa status [19].

\section{Pharyngeal phase}

Proper sealing of the nasopharynx by the velopharyngeal muscles and inversion of the epiglottis with suppression of respiratory movements protect the airway from food penetration during the pharyngeal phase of swallowing. In a case-control study, Bushmann et al. performed videofluoroscopy in 20 PD patients and 13 controls before and 45-90 min after the usual and individualized dose of levodopa. Swallowing impairment was detected in 15 patients and in 1 control. The most frequent abnormalities were delayed swallowing reflex and decreased laryngeal elevation. Five patients with swallowing impairment showed improvement in swallowing parameters following levodopa therapy; swallowing deteriorated in 1 patient. Four patients agreed to increase levodopa dosage and undergo repeat videofluoroscopy. Three did not show improvement; one had decreased valecular stasis and normalized laryngeal elevation [10]. When comparing dyskinetic and nondyskinetic PD patients with normal controls, nondyskinetic subjects displayed an increased frequency of pharyngeal stasis for thin liquids and solids, which was significantly related to clinical swallowing complaints [17].

\section{- Esophageal phase}

Although oropharyngeal dysphagia accounts for the majority of clinical symptoms, it is important to emphasize that poor upper esophageal sphincter (UES) relaxation, with or without incomplete opening, frequently may be present in PD patients. Ali et al. analyzed UES opening and relaxation by manometry in 19 PD patients and 23 healthy controls and reported that PD patients had reduced UES diameter and higher intrabolus pressure, compared with normal controls [20]. This abnormality can delay pharyngeal bolus transportation and increase the frequency of airway aspiration. Prolonged esophageal transit time and a tendency for retention in the lower portion of the esophagus may be present in PD patients, compared with controls, even in the absence of clinical symptoms. To date, however, no studies have evaluated the effect of levodopa on the esophageal phase of swallowing in PD patients.

\section{Conclusions}

Although several reports support the idea that levodopa therapy improves swallowing impairment in PD, there is no evidence that the drug consistently improves deglutition in individuals with PD. Since dopaminergic replacement may improve posture and respiration [21], some improvement in deglutition also might be expected; however, further controlled trials are needed to clarify this issue.

\section{Conflict of interests}

The authors declare no conflict of interest in this manuscript.

\section{References}

[1] Hamdy S, Aziz Q, Rothwell JC, Singh KD, Barlow J, Hughes DG, et al. The cortical topography of human swallowing musculature in health and disease. Nat Med 1996;2:1217-24.

[2] Fernandez HH, Lapane KL. Predictors of mortality among nursing home residents with a diagnosis of Parkinson's disease. Med Sci Monit 2002;8:CR241-6.

[3] Jean A. Brain stem control of swallowing: neuronal network and cellular mechanisms. Physiol Rev 2001;81:929-69.

[4] Cersosimo MG, Benarroch EE. Neural control of the gastrointestinal tract: implications for Parkinson disease. Mov Disord 2008;23:1065-175.

[5] Fontana GA, Pantaleo T, Lavorini F, Benvenuti F, Gangemi S. Defective motor control of coughing in Parkinson's disease. Am J Respir Crit Care Med 1998; 158:458-64.

[6] Ebihara S, Saito H, Kanda A, Nakajoh M, Takahashi H, Arai H, et al. Impaired efficacy of cough in patients with Parkinson disease. Chest 2003;124:1009-15.

[7] Rodrigues B, Nobrega AC, Sampaio M, Argolo N, Melo A. Silent saliva aspiration in Parkinson's disease. Mov Disord 2011;261:138-41.

[8] Gross RD, Atwood Jr CW, Ross SB, Eichhorn KA, Olszewski JW, Doyle PJ. The coordination of breathing and swallowing in Parkinson's disease. Dysphagia 2008;23:136-45.

[9] Nobrega AC, Rodrigues B, Melo A. Is silent aspiration a risk factor for respiratory infection in Parkinson's disease patients? Parkinsonism Relat Disord 2008; 14:646-8.

[10] Bushmann M, Dobmeyer SM, Leeker L, Perlmutter JS. Swallowing abnormalities and their response to treatment in Parkinson's disease. Neurology 1989; 39:1309-14.

[11] Kalf JG, de Swart BJ, Bloem BR, Munneke M. Prevalence of oropharyngeal dysphagia in Parkinson's disease: a meta-analysis. Parkinsonism Relat Disord 2012;18:311-5.

[12] Parkinson J. An essay on the shaking palsy. London: Sherwood, Neely and Jones; 1817 
[13] Calne DB, Shaw DG, Spiers AS, Stern GM. Swallowing in Parkinsonism. Br J Radiol 1970;43:456-7.

[14] Hunter PC, Crameri J, Austin S, Woodward MC, Hughes AJ. Response of parkinsonian swallowing dysfunction to dopaminergic stimulation. J Neurol Neurosurg Psychiatr 1997;63:579-83.

[15] Fuh JL, Lee RC, Wang SJ, Lin CH, Wang PN, Chiang JH, et al. Swallowing difficulty in Parkinson's disease. Clin Neurol Neurosurg 1997;99:106-12.

[16] Nagaya M, Kachi T, Yamada T, Igata A. Videofluorographic study of swallowing in Parkinson's disease. Dysphagia 1998;13:95-100.

[17] Monte FS, da Silva-Junior FP, Braga-Neto P, Nobre e Souza MA, de Bruin VM. Swallowing abnormalities and dyskinesia in Parkinson's disease. Mov Disord 2005;20:457-62.
[18] Umemoto G, Tsuboi Y, Kitashima A, Furuya H, Kikuta T. Impaired food transportation in Parkinson's disease related to lingual bradykinesia. Dysphagia 2011;26:250-5.

[19] Lim A, Leow L, Huckabee ML, Frampton C, Anderson T. A pilot study of respiration and swallowing integration in Parkinson's disease: "on" and "off" levodopa. Dysphagia 2008;23:76-81.

[20] Ali GN, Wallace KL, Schwartz R, DeCarle DJ, Zagami AS, Cook IJ. Mechanisms of oral-pharyngeal dysphagia in patients with Parkinson's disease. Gastroenterology 1996;110:383-92.

[21] Monteiro L, Souza-Machado A, Valderramas S, Melo A. The effect of levodopa on pulmonary function in Parkinson's disease: a systematic review and metaanalysis. Clin Ther 2012;34:1049-55. 